\title{
Managing the Elderly with Urinary Incontinence and Dementia
}

\section{Si Ching LIM*}

\section{Senior Consultant, Department of Geriatric Medicine, Changi General Hospital, Singapore}

*Corresponding author: Si Ching LIM, Senior Consultant, Department of Geriatric Medicine, Changi General Hospital, Singapore, E-mail: si_ching_lim@cgh.com.sg

\begin{abstract}
The elderly patients with dementia are often challenging to manage, especially if they have urinary incontinence. There are many causes for urinary incontinence and among the elderly with dementia, the problem is often not related to abnormalities of the lower urinary tract. Treatment options are limited by the multiple comorbidities, cognitive issues, medication side effects and limited efficacy among this group of frail elderlies.
\end{abstract}

\section{Keywords}

Elderly, Urinary incontinence, Dementia, Functional incontinence

\section{Introduction}

Urinary Incontinence (UI) is defined as involuntary leakage of urine. It is a common problem among the elderly, estimated to affect about $11-21 \%$ of community dwelling elderly in an Italian study and up to $77 \%$ of the residents in nursing homes $[1,2]$. Among the elderly with dementia, the prevalence is higher with $22 \%$ of the community dwelling elderly and $84 \%$ of nursing home residents reported to have UI [3]. Even though urinary incontinence is a common problem among the elderly, it should not be considered as synonymous with the normal ageing process. UI in the elderly is often due to a combination of factors arising from abnormalities of the lower urinary tract and factors not related to the urinary tract [4].

Urinary incontinence among the elderly with dementia is associated with caregiver burden and is a contributing factor for nursing home placement. $\mathrm{UI}$ is also associated with increased risk for pressure ulcers, falls, fractures, increased risk of urinary tract infections and cost. The presence of $\mathrm{UI}$ is associated with more severe cognitive impairment and more frequent reports of behavioural symptoms of dementia [5]. Despite this, the causes of UI among the elderly with dementia are not well studied because of multiple confounding factors like cognitive and physical disabilities.

This article reviews the common causes of $\mathrm{UI}$ among the elderly, especially the elderly with dementia and management strategies for UI among the elderly with dementia.

\section{Basic Neurophysiology of Micturition}

The bladder is innervated by the autonomic nerves as well as the somatic nerves. The sympathetic innervation originates at T11-L2, supplying the bladder via the Hypogastric nerve. The parasympathetic nerves originate at S2-S4 and innervate the bladder via the Pelvic and Pudendal nerves.

The sensation of bladder fullness as it fills ascends via the spinal cord to be conveyed to the Pontine Micturition Centre, which promotes micturition via the parasympathetic supply to the urinary bladder. However, micturition may not be appropriate at all times. The central nervous system determines the "correct timing" of micturition, taking into account the social and behavioural part of micturition. Micturition is coordinated and triggered through simultaneous activities of the bladder contraction with relaxation of the urethral sphincters via the somatic and autonomic nerves.

As the bladder fills, the sympathetic innervation promotes bladder wall relaxation and distension with efferent input to the urethral sphincters to cause sphincter contraction. The Frontal Micturition Centre, basal ganglia and the Pontine Micturition Centre actively inhibit

Citation: Si Ching LIM (2017) Managing the Elderly with Urinary Incontinence and Dementia. Int Arch Urol Complic 3:027. doi.org/10.23937/2469-5742/1510027

Received: April 14, 2017: Accepted: June 03, 2017: Published: June 05, 2017

Copyright: (C) 2017 Si Ching LIM. This is an open-access article distributed under the terms of the Creative Commons Attribution License, which permits unrestricted use, distribution, and reproduction in any medium, provided the original author and source are credited. 
detrusor contraction and augment urethral contraction during filling [6]. When the time is right for micturition, the parasympathetic nerves send efferent activating input to the bladder to promote contractions with input to the urethral sphincters causing relaxation of the sphincters. The sympathetic system is inhibited during voiding. During voiding, urethral sphincter activities ceases first followed by a rise in detrusor contraction and urine flows [7].

In addition to the complex neuroanatomical and physiological reflexes to maintain continence, there are other faculties needed to maintain continence. An intact cognition is important to perceive the sensation of bladder fullness with the ability to postpone micturition after the first sensation, motivation and desire to pass urine, sufficient mobility and coordination to reach the toilet with hand dexterity to manipulate clothing items in order to do so. In addition, the ability to locate the toilet with clear direction and access also contribute to continence [6].

\section{Physiological Changes Associated with the Ageing Lower Urinary Tract}

Urinary incontinence increases with rising age. Ageing is associated with changes in the lower urinary tract which predispose an elderly to UI. As we age, bladder capacity and contractility reduce, with reduced ability to defer voiding once the urge to do so arises. The post-void residual urine volume increases with age. During the storage phase, detrusor shows increased uninhibited contractility [8].

Among the elderly postmenopausal women, the pelvic muscles show loss of volume and tone. The ligamentous and connective tissue support for the pelvic organs gradually fail because of ageing. The weakened pelvic floor increases the risk of pelvic organ prolapse causing cystocele, rectocele and uterine prolapse. Stage 3-4 prolapse of pelvic organs can cause UI. A weakened pelvic floor also allows a hypermobile urethra to slide downwards during sudden increase in intraabdominal pressure [9].

Among the elderly men, it is estimated that histological evidence of Benign Prostatic Hyperplasia (BPH) is present in $90 \%$ of men by age 80 . Although BPH is synonymous with ageing, it does not correlate directly with prostatic enlargement. Lower Urinary Tract Symptoms
(LUTS) which consist of voiding and storage symptoms are common among the elderly men and increase in severity with age. Prostatic enlargement causes bladder outlet obstruction with voiding and storage symptoms. The association between LUTS and BPH in elderly men are temporal in onset but are not causally related. The differential diagnoses of LUTS in elderly men include urological and functional causes such as neurological disorders, Diabetes Mellitus, urethral stricture, etc. [10].

\section{Dementia and Changes to the Lower Urinary Tract}

Dementia is an umbrella of neurodegenerative disorders which cause degeneration of the CNS. The Alzheimer's Disease Association estimated that currently, there are 46.8 million people living with dementia worldwide and this number will double every 20 years to 74.7 million in 2030 and 131.5 million in 2050. Much of this increase will be in the developing world.

Central control of detrusor activities includes the frontal cortex, basal ganglia, Pontine Micturition Centre. The central control provides an inhibitory input on the detrusor to reduce contractions during the bladder filling phase. Dementia, particularly vascular dementia, normal pressure hydrocephalus, Frontotemporal lobe dementia, Alzheimer's Disease (AD) present with features of detrusor over-activity where urgency is the main symptom. Among the elderly with Alzheimer's disease, urge incontinence is the commonest presentation and UI is proportional to the dementia severity and impairment of ADLs [11].

Diffuse Lewy Body Dementia (DLBD), Multisystem atrophy, Parkinson's Disease Dementia (PDD) and AD have additional component of autonomic dysfunction, in addition to the central causes. The autonomic dysfunction presents with detrusor over-activity as the main type of UI. The synuclein deposition in PDD and DLBD is present in central nervous system and postganglionic sympathetic nerves [12].

\section{Types of Urinary Incontinence}

\section{Transient incontinence}

Urinary incontinence of recent onset, defined as four months' duration or less, is classified as transient incontinence. The causes are listed in Table 1 [13].

Table 1: Causes of Transient UI (DIAPPERS).

D- Delirium

Incontinence is a secondary feature and resolves once delirium improve. Common causes of delirium include acute medical conditions like stroke, infection, pain, fractures, medications, changes in environment, recent surgery, etc.

I- Symptomatic urinary infection Asymptomatic bacteriuria is common among the elderly and does not cause UI.

A- Atrophic vaginitis

P- Pharmacological Causes local irritability and contributes to UI.

Anticholinergics, diuretics, antidepressants, antipsychotics, sedatives/hypnotics, anti-Parkinson's treatment, etc.

P- Psychological

E- Excessive urine output Depression.

Diabetes Insipidus, excess fluid intake, diuretics- incl alcohol and caffeinated drinks, heart failure. Peripheral oedema. R- Restricted mobility Immobility due to restraint use, pain, lower limb arthritis, functional decline.

S- Stool impaction 
Table 2: Causes of Established UI.

\begin{tabular}{|c|c|c|c|c|}
\hline Causes & Detrusor over-activity & Stress incontinence & $\begin{array}{l}\text { Bladder outlet } \\
\text { obstruction }\end{array}$ & Detrusor underactivity \\
\hline Lower urinary tract & $\begin{array}{l}\text { Cystitis-infection/ } \\
\text { chemical, bladder stone, } \\
\text { tumour. } \\
\text { High urethral resistance. }\end{array}$ & $\begin{array}{l}\text { Pelvic floor weakness- } \\
\text { multiparity, obesity, } \\
\text { surgical procedures. } \\
\text { Post TURP in men. }\end{array}$ & $\begin{array}{l}\text { BPH, bladder neck } \\
\text { dysfunction, posterior } \\
\text { urethral valves, } \\
\text { iatrogenic-postop. }\end{array}$ & $\begin{array}{l}\text { Post-surgical complications } \\
\text { TURP, hip op, hysterectomy. } \\
\text { Pelvic organ prolapses, } \\
\text { severe atrophic vaginitis. } \\
\text { Anticholinergic drugs. }\end{array}$ \\
\hline Neurological causes & $\begin{array}{l}\text { DM neuropathy, spinal } \\
\text { cord lesions, Parkinson's } \\
\text { disease, MSA, stroke, } \\
\text { dementia. }\end{array}$ & $\begin{array}{l}\text { Pudendal nerve injury, } \\
\text { sacral spinal cord. }\end{array}$ & $\begin{array}{l}\text { Uncoordinated } \\
\text { sphincter activity- } \\
\text { Spinal cord injury. }\end{array}$ & $\begin{array}{l}\text { Multiple sclerosis, PD, DM, } \\
\text { dementia, sacral neuropathy, } \\
\text { stroke. }\end{array}$ \\
\hline
\end{tabular}

\section{Established urinary incontinence}

When the duration of $\mathrm{UI}$ is over 4 weeks and there are often abnormalities of the lower urinary tract. There are 4 types under this category: detrusor over-activity, stress incontinence, bladder outlet obstruction and detrusor underactivity. In some patients, it may be a combination of more than one type, called mixed incontinence.

Detrusor over-activity: Clinically, detrusor over-activity presents as urge incontinence, nocturia and frequency. Causes of detrusor over-activity are as listed in Table 2. Among the established causes of UI, detrusor over-activity is the commonest cause.

Variants of detrusor over-activity include concomitant detrusor hypocontractility present in half of the subjects with detrusor over-activity [14]. This condition is called Detrusor Hyperactivity with Impaired Contractility (DHIC). DHIC is associated with a high Post-Void Residual Volume (PVRU). The increase in PVRU is related to urethral resistance, rather than cortical abnormalities. Clinically, DHIC is problematic because treatment with anticholinergic agents for detrusor over-activity is contraindicated in the presence of high PVRU [15].

Urge incontinence with reduced bladder sensation presents with a sudden urgency to void with threat of incontinence, triggered by an involuntary detrusor contraction during filling in a bladder which is otherwise devoid of sensation. This is often associated with cognitive impairment and impaired cortical perfusion, suggestive of a cortical cause $[16,17]$.

Stress incontinence: Stress incontinence presents as involuntary leakage of urine with increase in intra-abdominal pressure such as laughing, sneezing, coughing or lifting. The causes are generally related to pelvic floor weakness causing loss of urethral support and opening of bladder neck. However, the maximum urethral closure pressure is by far the main predictor of stress incontinence [18]. Normally, a sudden increase in intra-abdominal pressure causes a rapid somatic response via the guarding reflex which activates simultaneous contraction of pelvic floor muscles and sphincter. If either of these is weak, the guarding reflex is ineffective [19].

Stress incontinence is the ccommonest type of $\mathrm{UI}$ among women. It is uncommon among men, except for cases post prostatectomy. Physical examination is often unremarkable. PVRU is not useful either because it is seldom significantly elevated without a concomitant neurological disorder, severe pelvic organ prolapses or recurrent Urinary Tract Infection (UTI) [20].

Bladder outlet obstruction: Bladder Outlet Obstruction (BOO) (causes in Table 2) is often associated with urge incontinence initially. The obstructed bladder develops hypertrophy and loss of parasympathetic nerve endings with disease progression. The hypertrophied bladder shows weaker detrusor contractions [21].

Detrusor underactivity: Detrusor Underactivity (DU) is common among patients with neurological diseases (Table 2). Risk factors for DU include drugs, prolonged untreated BOO, constipation, immobility, recurrent UTI and anaesthesia [22].

\section{Functional incontinence}

Functional $\mathrm{UI}$ is where there is no pathology in lower urinary tract or the micturition mechanism and is due to factors not directly related to lower urinary tract or micturition. Dementia cause deficits in memory, language, executive functioning, visuospatial function, loss of coordination, abnormal gait, behavioural symptoms, etc. Broadly, persons with dementia have difficulties with activities of daily living, behavioural symptoms and cognitive symptoms as dementia advances. For cognitive dysfunction, amnesia is the most prominent feature, aphasia presents with difficulties communicating with the caregivers for their needs, apraxia causes difficulties with familiar motor tasks such as dressing and using familiar household objects, agnosia presents as difficulty in recognising objects, people and places.

These deficits result in a person with difficulties recognising the sensation of bladder fullness, appropriateness to void, or to delay voiding until it is socially appropriate to do so. Among the elderly with dementia, lack of motivation to maintain dryness and failure to recognise the sensation of bladder fullness as a cue to head towards the toilet may be incontinent despite a normal lower urinary tract. They may have problems walking to and recognising the toilet, poor dexterity and inability to manipulate their clothing prior to voiding. In addition to cognitive symptoms, there are often behavioural symptoms where the elderly may present with agitation, restlessness and aggression because of 
the inabilities to toilet independently, communicate their toileting needs to the caregivers or discomfort due to high residual volume. As a result of agitation and restlessness, they may be physically restrained which worsens the UI. Mood disorders like depression and apathy are common symptoms of dementia which may present with poor motivation to maintain continence.

Unsteady gait with high falls risk is another common feature among the elderly with dementia. The subcortical dementias like vascular dementia, normal pressure hydrocephalus, Parkinson's disease dementia, frontotemporal dementia present with gait abnormalities early in the disease course. The unsteady gait predisposes to falls when the elderly with urgency rush for the toilet, and are often incontinent on their way to the toilet.

At the severe stages of dementia, the elderly is bed bound and uncommunicative. At this stage, most of them are on incontinent aids like diapers and catheters. This may lessen caregiver burden and reduce risk of pressure sores but increase risk of UTI [23,24].

Indwelling urinary catheters are commonly used in the elderly for various causes of UI. The long-term use of indwelling urinary catheters is discouraged. Long term indwelling urinary catheters are uncomfortable, known to increase risks of bladder stones and nephrolithiasis. Chronic indwelling catheter usage is known to have high risk for Urinary Tract Infections (UTI) like chronic pyelonephritis, symptomatic UTI with acute pyelonephritis and bacteraemia. UTIs in the presence of chronic indwelling catheters are often polymicrobial and resistant to single antibiotic therapy. Patients with indwelling catheters for more than 10 years' duration have been associated with bladder cancer and renal complications and these patients are advised to have regular follow-up renal scans, urine cytology and cystoscopy.

Indwelling catheters may be indicated for patients where skin dryness is important for pressure sore care, palliative care of patients where routine change of diapers or bed clothes cause severe discomfort, patients where $\mathrm{BOO}$ is not correctible medically or surgically or cases where it is the patient's preference to have an indwelling catheter as the incontinent aid [25].

\section{Managing the Elderly with UI and Dementia}

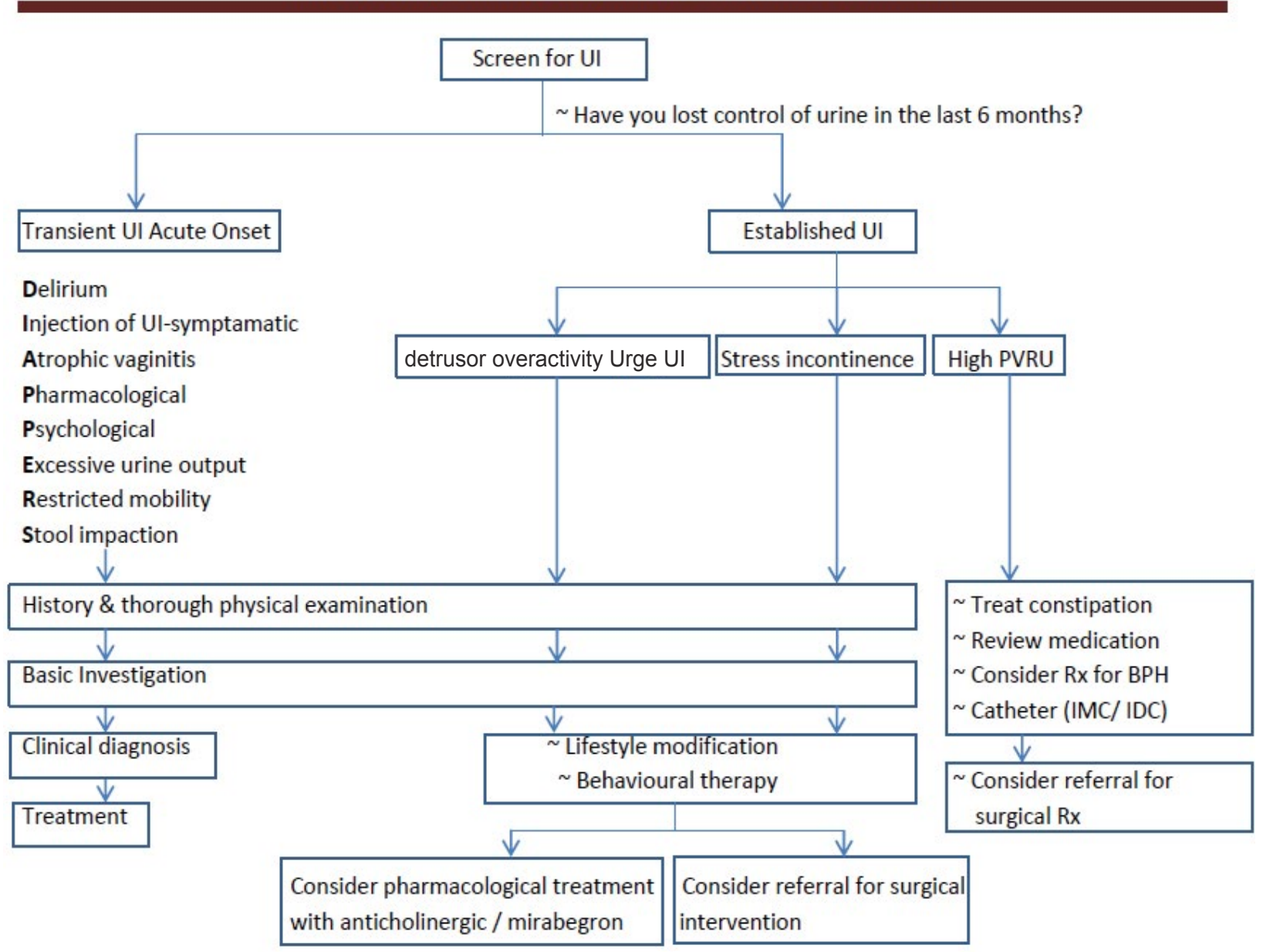

Ongoing Reassessment

Assess mobility, motivation to maintain continence, dexterity, accessibility to toilet, awareness for bladder fullness

Figure 1: Summary of management of UI. 


\section{Evaluation of UI}

Careful history taking and physical examination to exclude probable causes of transient UI (DIAPPERS) as listed in Table 1. Thorough physical examination should include a rectal examination for constipation which is often forgotten and is easily treatable. A bedside PVRU is helpful particularly among the elderly who are restless and agitated.

For the elderly with dementia, it is important to enquire about the awareness of bladder fullness and wetting habit. Passive wetting suggests delirium, advanced dementia, depression or apathy, with no motivation to maintain continence. An elderly with severe dementia who is bed bound may be nursed with incontinence pads for caregivers' convenience and pressure sore risk, hence passive wetting is a learned behaviour. The presence of behavioural symptoms of dementia and unstable gait may be contributing to UI.

Laboratory investigations include basic blood tests, urine analysis and culture, for transient UI. Further investigations include uroflow studies and Urodynamic Studies (UDS). A full UDS for the elderly with dementia is difficult to perform and is invasive. Therefore, it should be considered only if other treatment modalities failed and the results have direct influence on treatment choices and outcome [3,23].

\section{Management Strategies for UI among the Elderly with Dementia}

It is often important to ask about $\mathrm{UI}$ in the presence of caregivers, as $\mathrm{UI}$ is frequently not reported voluntarily by the caregivers. Many of the elderly who are frail and demented have other comorbidities and the aetiologies for UI are often multiple. Even though UI cannot be cured, it can be managed and contained with appropriate continence aids to achieve a social/acceptable continence (Figure 1) [26].

It is important to establish goals of treatment with the caregivers and the elderly with dementia. The goals include decrease in specific symptom burden, dryness or using less continence aids for protection, enable social activities to maintain/enhance quality of life, maintain the current place of residence and reduce caregiver burden [20].

From an elderly with dementia's point of view, treatment options are rather limited because of the impaired cognitive function and pharmacological treatment may cause worsening of the cognitive function. Treatment options for $\mathrm{UI}$ consist of non-pharmacological means (behavioural treatment) and pharmacological.

\section{Non-Pharmacological Management UI for the Elderly with Dementia}

\section{Behavioural interventions}

These interventions are not curative but safe and reversible. However, for the behavioural treatment to be efficacious, it requires active participation of a motivat- ed patient with support from caregivers. For the elderly with dementia, it is challenging for them to remember to do exercises, learning new techniques may take repetitive coaching and the caregivers may be frustrated with the elderly persons' inability to fully comply with the instructions given to them [27].

This treatment modality includes:

- Keeping a bladder diary, includes timing of voiding, amount voided, types of fluids ingested and whether the patient was able to reach toilet on time.

- Scheduled voiding involves toileting the elderly at fixed interval. There is no attempt to educate patient or reinforce behaviours. Scheduled voiding is helpful to reduce episodes of wetting, especially with reference to the bladder diary by pre-empting UI episodes.

- Prompted voiding where the elderly is prompted if they have the need to use the toilet, and allows the individual to request for toilet visits to reduce episodes of incontinence.

- Combined toileting with mobility and strengthening exercises into toileting routines. This may be helpful for the elderly who are not motivated to mobilise, using trips to toilet as opportunities to exercise and walk.

Persistence with these interventions is rarely maintained. Outcome measures are based on wet checks and not UI. There are no data on long term follow-up nor impact on caregivers and patients. Many studies excluded elderly who are unable to respond to one step command or had poor language abilities [28,29].

Bladder training: This is useful for patients with urgency and frequency to reduce frequency of micturition and to increase bladder capacity. This can be augmented by conscious pelvic floor muscle contractions, relaxation techniques and distraction to delay voiding by gradually increasing the time interval between voiding. Bladder training is effective in reducing incontinent episodes by up to $57 \%$ [30]. However, in an elderly with dementia, this may not be efficacious since delaying voiding may cause agitation and restlessness due to discomfort.

Pelvic floor exercises: Also, known as Kegel exercises and is effective for stress, urge and mixed incontinence. The main challenge is to correctly identify and isolate the correct muscle group for exercises. There are aids available to correctly identify the muscle groups like electrical stimulation, self-help books, biofeedback and verbal feedback while doing a vaginal examination. Treatment success is mainly determined by isolating the correct muscle groups and patient's motivation rather than the treatment method used for training $[20,31]$.

Lifestyle modifications: Modification of the amount of fluid, timing of fluid intake and the types of fluid can help with incontinence. Excessive intake of fluid can lead 
to a sudden bladder fullness, incontinence and nocturia. However, overzealous fluid restriction may increase risk of dehydration. Caffeine is both a diuretic and a bladder irritant causing increase in bladder pressure and detrusor irritability [20,32].

Obesity is associated with increased risk of $\mathrm{UI}$ in both men and women, with a $20-70 \%$ increase in risk of daily incontinence for each 5-unit increase in BMI. The increase in intra-abdominal visceral fat weakens the pelvic floor muscle support and innervation causing stress and mixed incontinence. Among the obese men, obesity is associated with BPH and LUTS. Obesity increases oestrogen to testosterone ratio by aromatisation of testosterone. Obesity causing obstructive sleep apnoea triggers atrial natriuretic peptide production which causes diuresis contributing to nocturia. Weight loss, either by conservative approach or bariatric surgery has been shown to reduce incontinence and LUTS in men and women $[33,34]$.

The overall outcomes of behavioural interventions are difficult to measure. The measures of quality of life may not be directly related to complete dryness. The frail elderly with dementia have other factors which cause $\mathrm{UI}$ and there is a need for new tools and approaches to assess patients' and caregivers' UI specific quality of life [35].

Catheterisation for detrusor under-activity: The main treatment strategy for a poorly contractile bladder is clean intermittent catheterisation. Drugs with parasympathetic activities are not widely used because of poor efficacy and poor side effect profile. New treatment modalities like neuromodulation, neurostimulation or reconstruction with muscle transposition have been explored but data is limited for the elderly [22].

\section{Pharmacological Rx of UI}

Drug treatment for established $\mathrm{UI}$ is mostly for detrusor over-activity and $\mathrm{BPH}$.

\section{Detrusor Over-Activity}

Anticholinergic drugs are the drugs of choice. The current available drugs include oxybutinin, tolterodine, solifenacin and trospium. Treatment with anticholinergic agents results in a small decrease in leakage, corresponding to half a leakage per day. The persistence with anticholinergic agents and compliance are both poor after a year, and worsen with older age. The reasons given for discontinuation are no observable effects, spontaneous improvement without medications, side effect profile and switch to another medication. The common side effects for anticholinergics include, dry mouth, constipation.

Cognitive decline among the elderly with dementia are of particular concern with anticholinergic agents. High dose Oxybutinin ( $20 \mathrm{mg}$ ) has been associated with cognitive impairment. Oxybutinin is highly lipophilic and neutral and it readily penetrates the Blood Brain Barrier (BBB). The newer anticholinergic agents like tolterodine and propiverine are less likely to do so. Trospium has the lowest affinity for the BBB. Cognitive effects are under-reported because they are clinically subtle, difficult to differentiate from baseline dementia, not actively asked for in trials or may have accounted for high dropout rates in trials. Treatment with anticholinergic agents should be limited, particularly among patients who have prescriptions for other drugs with anticholinergic properties [36-39].

For the elderly with AD prescribed Acetylcholinesterase Inhibitors (AChI) and anticholinergic agents in combination, Lu and Tune showed a significant worsening in MMSE after 2 years compared to those on AChl alone [40] Sink, et al. found a 50\% faster rate of functional decline in patients taking combination of AChl and anticholinergic agents (oxybutynin or tolterodine) compared to those on AChl alone, even though the ADAS-Cog scores were unchanged [41].

\section{$\mathrm{BPH}$}

As men age, the prostate increases in size which gradually causes worsening of lower urinary tract symptoms, decrease in peak urine flow and voided volume with a resulting increase risk of acute urinary retention, UTI and obstructive nephropathy. Current pharmacological treatment strategy includes $\alpha$-blockers like alfuzosin, doxazosin, tamsulosin and terazosin and $5 \alpha$-Reductase Inhibitors (5ARIs) like dutasteride and finasteride, either as single agent or combination therapy. Alpha-blockers have been shown to cause a reduction in symptom score by $10-20 \%$ but no reduction in risk of disease progression or long term complications. $5 \alpha$-reductase inhibitors cause a reduction in prostate volume by inhibiting conversion of testosterone in the prostate. 5ARIs have been shown to reduce serious long term effects of BPH like ARU, worsening LUTS and has a disease modifying effect [42]. Combination therapy with $\alpha$-blockers and 5ARIs has been shown to be more effective than single agent in reducing LUTS and overall disease progression, particularly among men with big prostate, more severe symptoms and higher PSA levels [43].

For the elderly with dementia, the main concern for using $\alpha$-blockers is postural hypotension which is often asymptomatic and increases falls risk. Postural hypotension is common among the elderly with multiple comorbidities, polypharmacy and autonomic dysfunction. The falls risk is high among the elderly with dementia because of abnormal gait, poor safety awareness and impulsiveness.

\section{Surgical Management of UI}

Data on surgical management of $\mathrm{UI}$ among the frail elderly is scarce. Outcome measures are often confounded by comorbidities and postoperative compli- 
cations among the elderly. Age related physiological changes like pelvic floor weakness and impaired bladder function also affect the success of surgical treatment. The elderly is at a higher risk of postop morbidity and mortality than the younger patients, and the elderly with dementia have a high risk of developing postop delirium. Recent recommendations by the American College of Surgeons and American Geriatric Society for preoperative assessment of the elderly patients include assessment of comorbidities with optimization, medication management, nutritional improvement, screening for frailty, cognitive impairment and function preoperatively $[44,45]$.

For the elderly men with $\mathrm{BPH}$, the current new techniques available include transurethral incision of prostate, transurethral vapourisation of prostate, laser prostatectomy and open prostatectomy (with laporoscopic and robotic prostatectomy). Emerging experimental techniques involving endoscopic heat generation using microwave thermotherapy, radiofrequency transurethral needle ablation, high intensity focused ultrasound, hot water thermotherapy and electromagnetic radiofrequency methods. Injectable techniques like transurethral injection with ethanol ablation and botulism toxin, mechanical devices with intraprostatic stents and urethral lift are also emerging. Despite these, none have shown significant improvement in the long term compared to TURP. The improvements are largely in shortened hospital stay and surgical morbidity. We therefore need to explore how does BPH contribute to bladder outlet obstruction and how much prostatic volume reduction is needed to relief LUTS [46].

For the elderly women with stress incontinence, options include injection of bulking agents at the proximal urethra, midurethral sling, colposuspension, transvaginal/retropubic/ transobturator tension-free vaginal tape are all recognized surgical interventions for stress UI. They are effective for women well selected to enroll in large trials with good gain in quality of life, with a risk of postop complications like infection. However, the more frail, disabled elderly with cognitive impairment have not been well studied [47].

\section{Conclusion}

$\mathrm{UI}$ is a common problem among the frail elderly with dementia and carries with it significant morbidity and caregiver burden. The causes are often multifactorial and treatment options are limited. Pharmacological treatment is limited and non-pharmacological options too, are limited among the elderly with dementia. The surgical options are often suboptimal and carries with it postop morbidity and mortality. Maintaining social dryness with quality of life as the main outcome should be the goal, rather than complete dryness.

\section{References}

1. Maggi S, Minicuci N, Langlois J, Pavan M, Enzi G, et al (2001) prevalence rate of urinary incontinence in communi- ty dwelling elderly individuals: The Veneto Study. J Gerontol A Biol Med Sci 56: 14-18.

2. Offermans MP, Du Moulin MF, Hamers JP, Dassen T, Halfens RJ (2009) Prevalence of urinary incontinence and associated risk factors in nursing home residents: A systematic Review. Neurourol Urodyn 28: 288-294.

3. Skelly J, Flint AJ (1995) Urinary incontinence associated with Dementia. J Am Geriatr Soc 43: 286-294.

4. Dubeau CE (2006) The aging lower urinary tract. J Urol 175: S11-S15.

5. Cassells C, Watt E (2003) The impact of incontinence on older spousal caregivers. J Adv Nurs 42: 607-616.

6. Sakakibara R, Uchiyama T, Yamanishi T, Kishi M (2008) Dementia and the lower urinary dysfunction: With a reference to anticholinergic use in the elderly population. Int $\mathrm{J}$ Urol 15: 778-788.

7. Badlani GH, Davila GW, Michel MC, Rosette JJMCH (2009) Continence: current concepts and treatment strategies.

8. Ouslander JG (1997) Aging and the lower urinary tract. Am J Med Sci 314: 214-218.

9. Doumouchtsis SK, Chrysanthopoulou EL (2013) Urogenital consequences in ageing women. Best Pract Res Clin Obstet Gynaecol 27: 699-714.

10. Herbert lepor (2005) Pathophysiology of Lower Urinary Tract Symptoms in the ageing male population. Rev Urol 7: S3-S11.

11. Lee SH, Cho ST, Na HR, Ko SB, Park MH (2014) urinary Incontinence in patients with Alzheimer's Disease: Relationship between symptom status and urodynamic diagnosis. Int J Urol 21: 683-687.

12. Idiaquez J, Roman GC (2011) Autonomic dysfunction in neurodegenerative dementias. J Neurol Sci 305: 22-27.

13. Resnick NM (1995) Urinary incontinence. Lancet 346: 9499.

14. Resnick NM, Yalla SV, Laurino E (1989) the pathophysiology of urinary incontinence among institutionalised elderly persons. N Engl J Med 320: 1-7.

15. Griffiths DJ, McCracken PN, Harrison GM, Gormley EA, Moore KN (2002) Urge incontinence and impaired detrusor contractility in the elderly. Neurourol Urodyn 21: 126-131.

16. Geirsson G, Fall M, Lindström S (1993) Subtypes of overactive bladder in old age. Age Ageing 22: 125-131.

17. Griffiths DJ, McCracken PN, Harrison GM, Moore KN (1996) Urinary incontinence in elderly people: factors predicting the severity of urine loss before and after pharmacological treatment. Neurourol Urodyn 15: 53-57.

18. DeLancey JO, Trowbridge ER, Miller JM, Morgan DM, Guire K, et al. (2008) Stress urinary incontinence: relative importance of urethral support and urethral closure pressure. J Urol 179: 2286-2290.

19. Karen L Miller (2005) Stress Urinary Incontinence in Women: Review and Update on Neurological Control. J Women's Health (Larchmt) 14: 595-608.

20. Goode PS, Burgio KL, Richter HE, Markland AD (2010) Incontinence in older women. JAMA 303: 2172-2181.

21. Jacek L Mostwin (2002) Pathophysiology: the varieties of bladder overactivity. Urology 600: 22-26.

22. van Koeveringe GA, Vahabi B, Andersson KE, Kirschner-Herrmans R, Oelke M (2011) Detrusor underactivity: A plea for 
new approaches to a common bladder dysfunction. Neurouro Urodyn 30: 723-728.

23. Yap $P$, Tan D (2006) Urinary incontinence in dementia - a practical approach. Aust Fam Physician 35: 237-241.

24. Lim SC, Poon WH (2016) Restraint use in the management of the elderly with dementia in hospital. Internal Med Res Open J 1: 1-4.

25. Cravens DD, Zweig S (2000) Urinary catheter management. Am Fam Physician 61: 369-376.

26. DuBeau CE (2001) Urinary incontinence management: new questions from old assumptions. J Am Geriatr Soc 49: 829-830.

27. Wagg A, Gibson W, Ostaszkiewicz J, Johnson T, Markland A, et al. (2015) Urinary incontinence in frail elderly persons: Report from the 5th International Consultation on Incontinence. Neurourol Urodyn 34: 398-406.

28. Colling J, Owen TR, McCreedy M, Newman D (2003) The effects of a continence program on frail community-dwelling elderly persons. Urol Nurs 23: 117-122.

29. Engberg S, Sereika SM, McDowell BJ, Weber E, Brodak I (2002) Effectiveness of prompted voiding in treating urinary incontinence in cognitively impaired homebound older adults. J Wound Ostomy Continence Nurs 29: 252-265.

30. Fantl JA, Wyman JF, McClish DK, Harkins SW, Elswick RK, et al. (1991) Efficacy of bladder training in older women with urinary incontinence. JAMA 265: 609-613.

31. Goode PS, Burgio KL, Locher JL, Roth DL, Umlauf MG, et al. (2003) Effect of behavioral training with or without pelvic floor electrical stimulation on stress incontinence in women: a randomized controlled trial. JAMA 290: 345-352.

32. Arya LA, Myers DL, Jackson ND (2000) Dietary caffeine intake and the risk for detrusor instability: a case-control study. Obstet Gynecol 96: 85-89.

33. Whitcomb EL, Subak LL (2011) Effect of weight loss on urinary incontinence in women. Open Access J Urol 3: 123132.

34. Luke S, Addison B, Broughton K, Masters J, Stubbs R, et al. (2015) effects of bariatric surgery on untreated lower urinary tract symptoms: a prospective multicentre cohort study. BJU Int 115: 466-472.

35. Dubeau CE, Simon SE, Morris JN (2006) The effect of urinary incontinence on quality of life in older nursing home residents. J Am Geriatr Soc 54: 1325-1333.
36. Samuelsson E, Odeberg J, Stenzelius K, Molander U, Hammarström M, et al. (2015) Effect of pharmacological treatment for urinary incontinence and frail elderly. Geriatr Gerontol Int 15: 521-534.

37. Whalley LJ, Sharma S, Fox HC, Murray AD, Staff RT, et al. (2012) Anticholinergic drugs in late life: adverse effects on cognition but not on progress to dementia. J Alzheimers Dis 30: 253-261.

38. Fox C, Livingston G, Maidment ID, Coulton S, Smithard DG, et al. (2011) The impact of anticholinergic burden in Alzheimer's dementia-the LASER-AD study. Age Ageing 40: 730-735.

39. Fox C, Richardson K, Maidment ID, Savva GM, Matthews FE, et al. (2011) Anticholinergic medication use and cognitive impairment in the older population: The medical research council cognitive function and ageing study. J Am Geriatr Soc 59: 1477-1483.

40. Lu CJ, Tune LE (2003) Chronic exposure to anticholinergic medications adversely affects the course of Alzheimer disease. Am J Geriatr Psychiatry 11: 458-461.

41. Sink KM, Thomas J 3rd, Xu H, Craig B, Kritchevsky S, et al. (2008) Dual use of bladder anticholinergics and cholinesterase inhibitors: Long-term functional and cognitive outcomes. J Am Geriatr Soc 56: 847-853.

42. Naslund MJ, Miner M (2007) A review of the clinical efficacy and safety of $5 a-R e d u c t a s e$ Inhibitors for the enlarged prostate. Clin Ther 29: 17-25.

43. McVary KT (2007) A review of combination therapy in patients with Benign Prostatic Hyperplasia. Clin Ther 29: 387-389.

44. Sung VW, Weitzen S, Sokol ER, Rardin CR, Myers DL (2006) Effect of patient age on increasing morbidity and mortality following urogynecologic surgery. Am J Obstet Gynecol 194: 1411-1417.

45. Chow WB, Rosenthal RA, Merkow RP, Ko CY, Esnaola NF, et al. (2012) Optimal preoperative assessment of the geriatric surgical patient: a best practices guideline from the American College of Surgeons National Surgical Quality Improvement Program and the American Geriatrics Society. J Am Coll Surg 215: 453-466.

46. Thiruchelvam N (2014) Surgical therapy for benign prostatic hyperplasia/ bladder outflow obstruction. Indian J Urol 30: 202-207.

47. Dmochowski RR, Blaivas JM, Gormley EA, Juma S, Karram MM, et al. (2010) Update of AUA guideline on the surgical management of female stress urinary incontinence. $J$ Urol 183: 1906-1914. 